\title{
Feature Extraction and Identification in Distributed Optical-Fiber Vibration Sensing System for Oil Pipeline Safety Monitoring
}

\author{
Huijuan WU*, Ya QIAN, Wei ZHANG, and Chenghao TANG \\ Key Laboratory of Optical Fiber Sensing and Communications, Ministry of Education, University of Electronic Science \\ and Technology of China, Chengdu, 611731, China \\ ${ }^{*}$ Corresponding author: Huijuan WU_ E-mail: hjwu@uestc.edu.cn
}

\begin{abstract}
High sensitivity of a distributed optical-fiber vibration sensing (DOVS) system based on the phase-sensitivity optical time domain reflectometry ( $\Phi-O T D R)$ technology also brings in high nuisance alarm rates (NARs) in real applications. In this paper, feature extraction methods of wavelet decomposition (WD) and wavelet packet decomposition (WPD) are comparatively studied for three typical field testing signals, and an artificial neural network (ANN) is built for the event identification. The comparison results prove that the WPD performs a little better than the WD for the DOVS signal analysis and identification in oil pipeline safety monitoring. The identification rate can be improved up to $94.4 \%$, and the nuisance alarm rate can be effectively controlled as low as $5.6 \%$ for the identification network with the wavelet packet energy distribution features.
\end{abstract}

Keywords: Distributed optical-fiber vibration sensing; $\Phi$-OTDR; pattern recognition; multi-scale analysis

Citation: Huijuan WU, Ya QIAN, Wei ZHANG, and Chenghao TANG, "Feature Extraction and Identification in Distributed Optical-Fiber Vibration Sensing System for Oil Pipeline Safety Monitoring,” Photonic Sensors, 2017, 7(4): 305-310.

\section{Introduction}

The distributed optical-fiber vibration sensing (DOVS) system based on the phase-sensitivity optical time domain reflectometry ( technology provides a convenient and cost-effective disturbance detection and location method for safety monitoring of ultra-long distance perimeters, oil or gas pipelines, submarine or buried telecommunication cables, power transmission cables, and large structures [1]. However, the high sensitivity of the system induces that it is liable to be interfered by complicated disturbing sources in practical long-distance monitoring applications, which may result in a high nuisance alarm rate
(NAR) [5]. The conventional detection method for DOVS is always carried out by only differentiating the obtained OTDR traces before and after the intrusion occurrence [7]. And most of the related signal processing work focused on the improvement of detection, and very few mentioned the identification of the perturbing events except some frequency spectrum analysis with the fast Fourier transform (FFT) [10]. Due to the unpredictable influences of traffics, and other production and people's life interferences along the long monitoring line, the identification of the detected events is always a persisting predicament, which restricts its practical applications.

Thus, identifiable feature extraction methods for

Received: 14 June 2016 / Revised: 11 August 2017

(C) The Author(s) 2017. This article is published with open access at Springerlink.com

DOI: $10.1007 / \mathrm{s} 13320-017-0360-1$

Article type: Regular 
DOVS signals are comparatively studied in this paper to improve the perturbing event identification performance: energy spectrum distributions are extracted by using the wavelet decomposition (WD) and the wavelet packet decomposition (WPD) respectively for the varied types of disturbing event signals along the buried oil pipeline of about $65 \mathrm{~km}$ in practical field applications. Then we construct a 4-layer artificial neural network (ANN) to identify three typical events, background noises, artificial digging events, and vehicle passing interferences as a comparison of these two feature extraction methods. The practical application results show that the probability for correct detection has been improved considerably and NAR has been reduced effectively.

\section{Pipeline safety monitoring system based on DOVS with $\Phi$-OTDR technology}

The schematic diagram of pipeline safety monitoring system based on DOVS with $\Phi$-OTDR technology is shown in Fig. 1. A continuous coherent light generated from the ultra-narrow line-width laser is modulated to an optical pulse signal by the acoustic-optical-modulator (AOM). The optical pulse signal, amplified by the erbium-doped fiber amplifier (EDFA), is then gated into the sensing fiber cable through the optical isolator. The backscattered Rayleigh light is filtered by the optical filter to eliminate noises. A coherent optical time domain reflection signal, namely an OTDR track, is obtained after a photoelectric conversion by the photo-detector (PD) and then converted into a digital signal by the analog-digital-converter (ADC). The sensing fiber cable is usually an optical communication cable that has already been buried along the oil pipeline. One end of a spare fiber core is connected into the $\Phi-\mathrm{OTDR}$ demodulator. When an intrusion occurs near the pipeline, its vibration may generate phase disturbances in the closed fiber, which can be revealed from the received OTDR traces. Finally, the acquired data are processed in a processing unit to obtain and demonstrate the abnormal event detection and location results in real time.

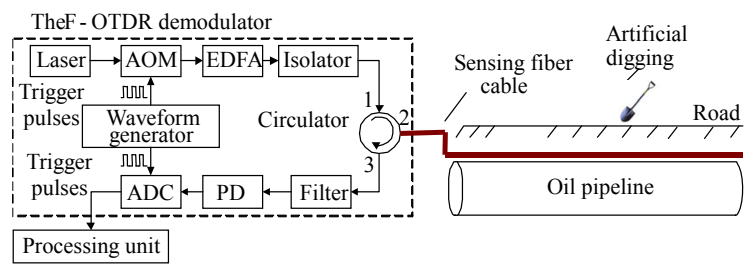

Fig. 1 Pipeline safety monitoring system.

The temporal sequence obtained along the time axis at each spatial point exactly demonstrates the different action modes of the different individual disturbing events, which can be obtained by accumulating the OTDR traces at different moments. Further, different types of event signals have different frequency components, thus they could have different concentrated distributions in the frequency domain. Therefore, the extracted energy distribution of different types of event signals by using multi-scale WD or WPD methods could be identifiable and distinguished from each other.

\section{Feature extraction and identification methods}

\subsection{Identifiable feature extraction based on multi-scale analysis}

WD [6] is processed like an unbalanced tree from top to bottom as shown in Fig. 2(a). The signal passing through the filter banks is decomposed into the approximations from low-pass filter and the details from the high-pass filter. With a $J$-level decomposition, the obtained details at each level are denoted as $\left[d_{1}, d_{2}, \cdots, d_{J}\right]$, and the approximations at $J$ th level is denoted as $a_{J}$. And we use $d_{(J+1)}$ to replace $a_{J}$ for uniform expression. The decomposed signals are sorted from low frequency components to high frequency components, which are denoted as $\left[s_{0}, s_{2}, \cdots, s_{j}\right]$. Then the corresponding energy of each decomposed frequency band is computed as 
follows:

$$
E_{w j}=\sum_{n=1}^{N}\left|d_{j}(n)\right|^{2}(j=1,2, \cdots, J, J+1)
$$

where $n$ is the index, and $N$ is the length of the sequence. By normalization, the wavelet energy (WE) vector in (1) is obtained as follows:

$$
T_{w}=\frac{1}{E_{w s u m}}\left[E_{w(J+1)}, E_{w J}, \cdots, E_{w 1}\right]
$$

where $E_{\text {wsum }}$ is the total sum of all the members of $E_{w j}$.

The WPD [11] applies the decomposition not only to the approximations but also to the details, offering richer frequency analysis than the WD. And the WPD process like a balanced tree from top to bottom is comparatively illustrated in Fig. 2(b). With a $j$-level decomposition, the WPD generates $2^{j}$ different decomposition components, which are denoted as $C_{j, l} l=0,1, \cdots, 2^{j}-1$ and $l$ is the terminal node index at the $j$ th level. The decomposed signals are sorted in the ascending order of frequency bands, which are denoted as $\left[\begin{array}{lll}\mathrm{s} 0 & \ldots & \mathrm{s} m\end{array}\right] \quad\left(m=2^{j}-1\right)$. The corresponding energy of each frequency band is obtained as

$$
E_{l}=\sum_{n=1}^{N}\left|C_{j, l}(n)\right|^{2}\left(l=0,1, \cdots, 2^{j}-1\right)
$$

where $n$ is the index, and $N$ is the length of the sequence. By normalization, the wavelet packet energy (WPE) vector in (3) is obtained as

$$
T_{w p}=\frac{1}{E_{w p s u m}}\left[E_{0}, E_{1}, \cdots, E_{m}\right]\left(m=2^{j}-1\right)
$$

where $E_{\text {wpsum }}$ is the total sum of all the members of $E_{l}$.

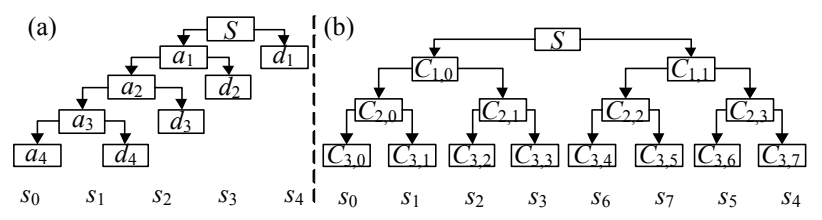

Fig. 2 Multi-scale decomposition tree: (a) wavelet decomposition and (b) wavelet packet decomposition.

\subsection{Identification network construction}

After the multi-scale analysis, the energy distributions of different event signals can be obtained. We also construct a 4-layer back propagation (BP) ANN network as shown in Fig. 3 for further event identification and determination. In the identification network, there is one input layer, two hidden layers, and one output layer. In the input layer, taking WE, or WPE or other feature vector as the basic input feature, the input knot number of the network is determined by the dimension of the feature vector and equals $2^{j}$. For the two hidden layers, generally a hyperbolic tangent sigmoid function is chosen as the activation function where five knots are set in the first hidden layer and three knots are set in the second hidden layer in this case according to series of experimental parameters test. The output layer with a linear activation function consists of two knots, which is decided by the target number. With this designed configuration, samples of three typical event signals in oil pipeline safety monitoring, such as artificial digging events, vehicle passing interferences, or just background noises, are input to the identification network to be trained to obtain suitable network parameters. A proper error threshold is set to stop the training process. The error threshold is a tradeoff between the identification precision and the computation cost. And for the test, the normalized WE/WPE of testing signals can be taken as the input to intelligently reveal if the vibration disturbing signal is caused by artificial digging events, vehicle passing interferences, or just background noises.

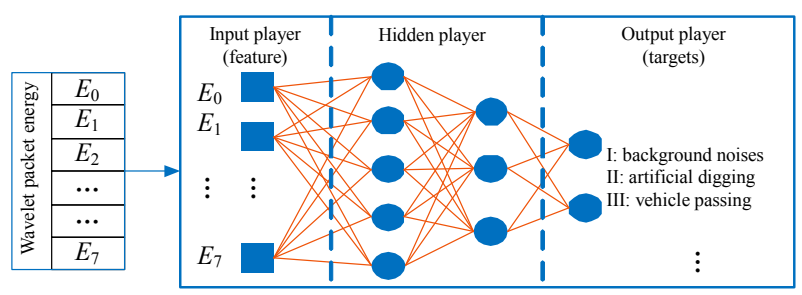

Fig. 3 4-layer BP ANN network for classification.

\section{Field test results and discussion}

\subsection{Energy distribution results}

In the field test, three typical event signals along a $65-\mathrm{km}$-long oil pipeline are obtained and analyzed, 
including background noises with no intrusion, artificial digging events, and vehicle passing interferences. The sampling rate of the accumulated temporal signal is $508 \mathrm{~Hz}$. The temporal signals of the three typical events in one second and their WE and WPE distributions are demonstrated in Figs. 4, 5, and 6 , respectively.
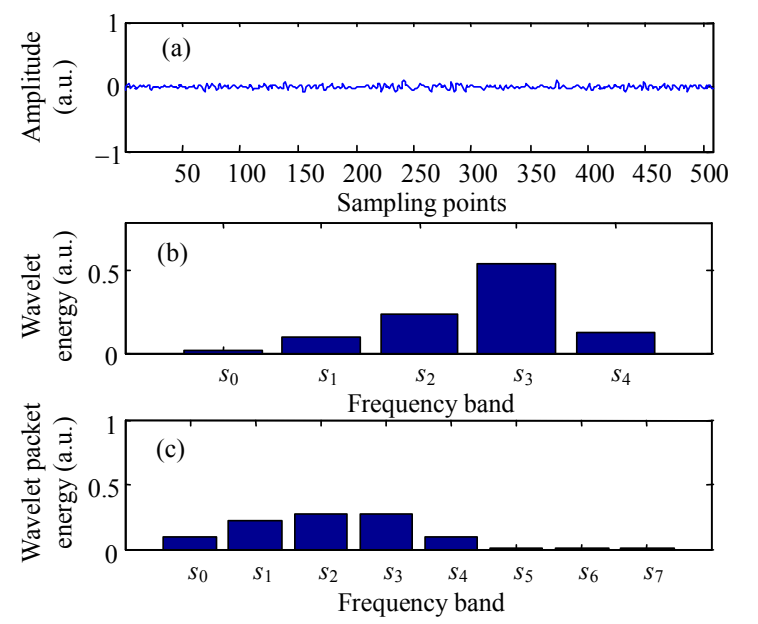

Fig. 4 Background noise signal and its WE and WPE distributions: (a) background noise signal, (b) wavelet energy distribution, and (c) wavelet packet energy distribution.
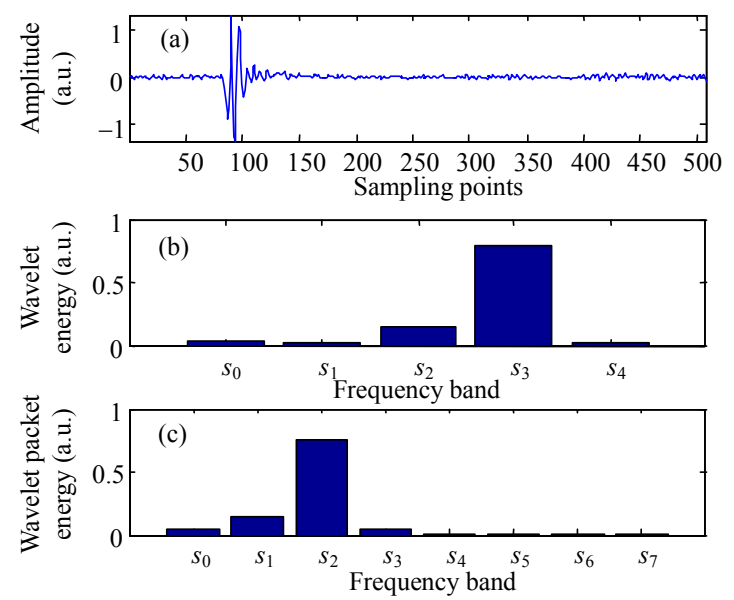

Fig. 5 Artificial digging signal and its WE and WPE distributions: (a) artificial digging signal, (b) wavelet energy distribution, and (c) wavelet packet energy distribution.

As shown in Figs. 4(b) and 5(b), for background noise and artificial digging event signals, the high frequency band $s_{3}$ dominates in both the two WE energy graphs, and the shapes of the WE distribution envelopes are similar. There is also difference in the two graphs: the bar of band $s_{3}$ is much higher than that of band $s_{2}$ for artificial digging signal; on the contrary, the bar height difference is less for background noise signal. However, the WPE can obtain more different distribution trends for all of the three types of event signals as shown in Figs. 4(c), 5(c), and 6(c). That's because the WD decomposes the signal frequency band with an uneven scale at different levels, which thus induces that the energies are always concentrated on a wider band of higher frequencies. As a result, it is not an ideal tool to evaluate the energy distribution difference in the frequency domain.
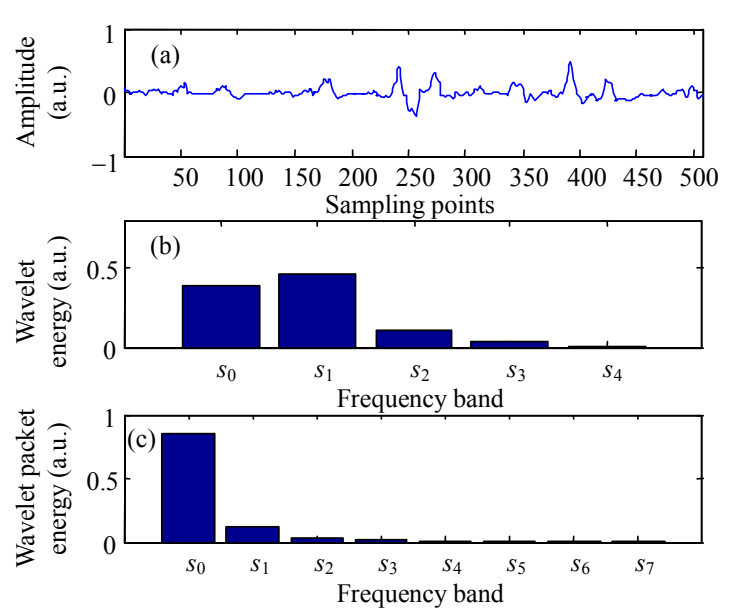

Fig. 6 Vehicle passing signal and its WE and WPE distributions: (a) vehicle passing signal, (b) wavelet energy distribution, and (c) wavelet packet energy distribution.

To further investigate the difference of these two decomposition methods in practical complex scenarios, more signal samples of the above three types of events are tested in a similar way, and the WE and WPE distributions of the tested samples are comparatively demonstrated in Figs. 7 and 8. In Fig. 7 , even though the vehicle passing interferences have typically different WE distribution trends, the distribution trends of the other two events could be similar, in which two of the three WE distributions of the artificial digging events (artificial digging II and III) nearly overlap with the WE distributions of the two background noises (background noise I and II). This conclusion agrees with the conclusion of the single case in Figs. 4 and 5. However, the WPE in Fig. 8 can demonstrate three typically different energy distribution trends for these three events. It is 
seen that the background noises always have a flat energy spectrum, the artificial digging signals always denominate the band $s_{1}$ and $s_{2}$ with higher frequencies in the low frequency components, and the vehicle passing events are always denominating the band $\mathrm{s} 0$ with the lowest frequencies. It can be summed up that the WPD is a better frequency spectrum analysis way which can obtain a more accurate frequency band decomposition.

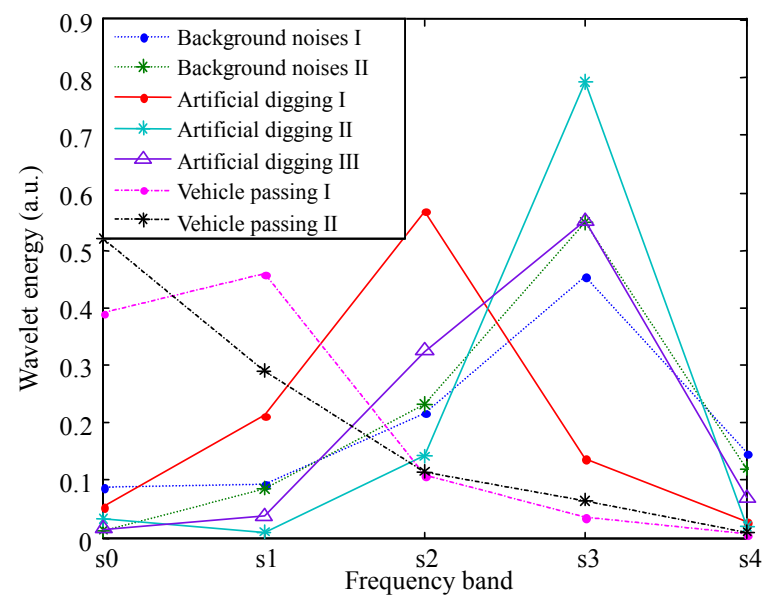

Fig. 7 WE distribution for three typical events.

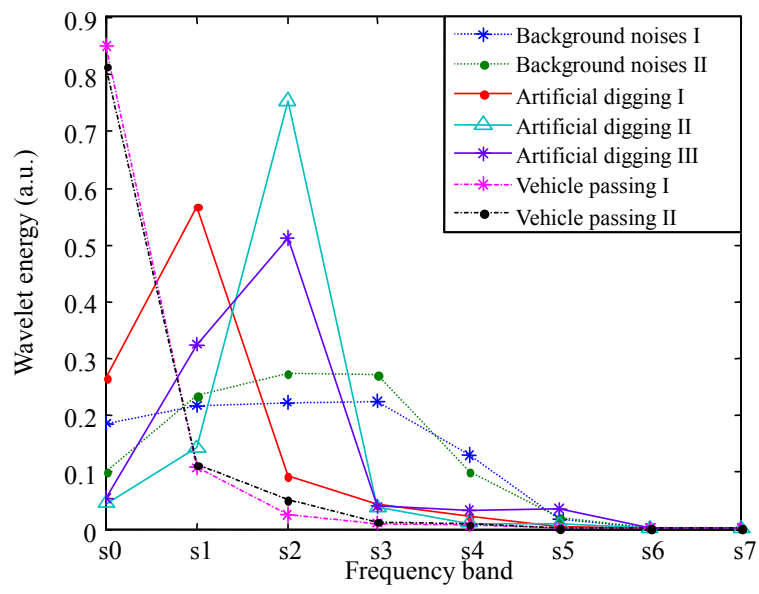

Fig. 8 WPE distribution for three typical events.

\subsection{Identification results}

To evaluate the feasibility of the proposed WD and WPD's feature extraction method, a database of 127 sample signals including 40 background noises, 47 artificial digging events, and 40 vehicle passing interferences, are first prepared to train the 4-layer ANN network as shown in Fig. 3. And the normalized WE and WPE $\left[E_{0}, E_{1}, \cdots, E_{7}\right]$ in decomposed frequency $\left[s_{0}, s_{1}, \cdots, s_{7}\right]$ of each sample event signal are respectively taken as the input vector at the input layer.

After well training, 90 field testing signals are inputted into the network, which contains 30 background noises, 30 artificial digging events, and 30 vehicle passing interferences. For comparison, the extracted WE and WPE feature are in turn input into the 4-layer BP network. And the identification results for the two feature extraction methods are concluded in Tables 1 and 2. The identification rate (IR) for all of the three events can reach higher than $90 \%$ for both methods. However, the identification results by using the features extracted by WPD in Table 2 are better than those by using the features extracted by WD in Table 1 . The average IR by using WPE can be achieved up to $94.4 \%$, and the overall NAR can be effectively controlled as low as $5.6 \%$, while for the results by using WE, the IR can be achieved to $91.1 \%$, and the NAR can be $8.9 \%$.

Table 1 Identification results by using WE.

\begin{tabular}{cccccccc}
\hline & I & II & III & Others & IR & $\begin{array}{c}\text { IR in } \\
\text { average }\end{array}$ & NAR \\
\hline I & 27 & 3 & 0 & 0 & $90.0 \%$ & & \\
II & 2 & 28 & 0 & 0 & $93.3 \%$ & $91.1 \%$ & $8.9 \%$ \\
III & 0 & 3 & 27 & 0 & $90.0 \%$ & & \\
\hline
\end{tabular}

I: Type I, background noises;

II: Type II, artificial digging events;

III: Type III, vehicle passing interferences;

Others: Unidentified events;

IR: Identification rate;

NAR: Nuisance alarm rate.

Table 2 Identification results by using WPE.

\begin{tabular}{cccccccc}
\hline & I & II & III & Others & IR & $\begin{array}{c}\text { IR in } \\
\text { average }\end{array}$ & NAR \\
\hline I & 27 & 2 & 0 & 1 & $90.0 \%$ & & \\
II & 2 & 28 & 0 & 0 & $93.3 \%$ & $94.4 \%$ & $5.6 \%$ \\
III & 0 & 0 & 30 & 0 & $100 \%$ & & \\
\hline
\end{tabular}

\section{Conclusions}

In this paper, feature extraction methods with WD and WPD are first comparatively studied for the DOVS signals in oil pipeline safety monitoring applications. And then the field testing results based on the BP network prove the effectiveness of WD 
and WPD as an identifiable feature extraction tool for the practical event signal classification, and WPD could be better for the identifiable feature extraction than WD. Therefore, it is promising to be practically applied in the oil pipeline safety monitoring system for pre-warning of the third-party damage.

\section{Acknowledgement}

The authors gratefully acknowledge the supports provided for this research by Youth Foundation (Grant No. 61301275), Major Instrument Special Program (Grant No. 41527805), the Major Program (Grant No. 61290312) of the National Science Foundation of China (NSFC), and the fund of State Grid Corporation of China: Research on distributed multi-parameter sensing and measurement control technology for electric power optical fiber communication networks (Grant No. 5455HT160014). This work is also supported by Program for Changjiang Scholars and Innovative Research Team in University (PCSIRT, IRT1218) and the 111 Project (B14039).

Open Access This article is distributed under the terms of the Creative Commons Attribution 4.0 International License (http://creativecommons.org/ licenses/by/4.0/), which permits unrestricted use, distribution, and reproduction in any medium, provided you give appropriate credit to the original author(s) and the source, provide a link to the Creative Commons license, and indicate if changes were made.

\section{References}

[1] Y. J. Rao, J. Luo, Z, L. Ran, J. F. Yue, X. D. Luo, and Z. Zhou, "Long-distance fiber-optic $\Phi-O T D R$ intrusion sensing system," SPIE, 2009, 7503: 1-4.

[2] W. T. Lin, S. Q. Lou, and S. Liang, "Fiber-optic distributed vibration sensor for pipeline pre-alarm," Applied Mechanics and Materials, 2014, 684: 235-239.
[3] H. J. Wu, Y. Qian, H. Y. Li, S. K. Xiao, Z. Z. Fu, and Y. J. Rao, "Safety monitoring of long distance power transmission cables and oil pipelines with OTDR technology," in Proceeding of Laser and Electro-optics (CLEO), San Jose, CA, USA, 2015, pp: $1-2$.

[4] F. Peng, H. Wu, X. H. Jia, and Z. P. Peng, "Ultra-long high-sensitivity $\Phi-O T D R$ for high spatial resolution intrusion detection of pipelines," Optics Express, 2014, 22(11): 13804-13810.

[5] H. J. Wu, X. Y. Li, Z. P. Peng, and Y. J. Rao, "A novel intrusion signal processing method for phase-sensitive optical time-domain reflectometry (Ф-OTDR)," SPIE, 2014, 9157: 9157O-1-9157O-4.

[6] H. J. Wu, S. K. Xiao, X. Y. Li, Z. N. Wang, J. W. Xu, and Y. J. Rao, "Separation and determination of the disturbing signals in phase-sensitive optical time domain reflectometry (Ф-OTDR)," Journal of Lightwave Technology, 2015, 33(15): 3156-3162.

[7] Z. G. Qin, L. Chen, and X. Y. Bao, "Wavelet denoising method for improving detection performance of distributed vibration sensor," IEEE Photonics Technology Letters, 2012, 24(7): 542-544.

[8] Q. Li, C. X. Zhang, and C. S. Li, "Fiber-optic distributed sensor based on phase-sensitive OTDR and wavelet packet transform for multiple disturbances location," Optik, 2014, 125(24): 7235-7238.

[9] H. M. Yue, B. Zhang, Y. X. Wu, B. Y. Zhao, J. F. Li, J. H. Ou, et al., "Simultaneous and signal-to-noise ratio enhancement extraction of vibration location and frequency information in phase-sensitive optical time domain reflectometry distributed sensing system," Optical Engineering, 2015, 54(4): 047101-1047101-6.

[10] Z. Y. Wang, Z. Q. Pan, Q. Y. Qing, B. Lu, Z. J. Fang, H. W. Cai, et al., "Novel distributed passive vehicle tracking technology using phase sensitive optical time domain reflectometer," Chinese Optics Letters, 2015, 13(10): 30-34.

[11] B. Asgarian, V. Aghaeidoost, and H. R. Shokrgozar, "Damage detection of jacket type offshore platforms using rate of signal energy using wavelet packet transform," Marine Structures, 2015, 45: 1-21.

[12] A. B. Meng, J. F. Ge, H. Yin, and S. Z. Chen, "Wind speed forecasting based on wavelet packet decomposition and artificial neural networks trained by crisscross optimization algorithm," Energy Conversion and Management, 2016, 114: 75-88. 